\title{
Urinary toluene levels and adverse health symptoms among automotive garage workers, Nakhon Si Thammarat province, Thailand
}

\author{
Somsiri Decharat ${ }^{1, *}$ (iD) \\ ${ }^{1}$ Department of Occupational health and Safety, Faculty of Health and Sports Science, Thaksin University, 222 Papayom \\ District, Phattalung Province 93210, Thailand \\ *Correspondence: somsiri_9@hotmail.com
}

Received: May 12, 2021 Accepted: July 5, 2021

\begin{abstract}
To determine their urinary toluene levels, to describe the workers' hygiene behaviors and the prevalence of adverse health symptoms among automotive garage workers exposed to chemical substances. A cross-sectional descriptive study was conducted by interviewing among automotive garages located in the Nakhon Si Thammarat province, Thailand. During between 1 November 2020 and 31 December 2020. A total of 140 automotive garages workers were selected using a purposive sampling method. The questionnaire was conducted via face-to-face interview and the toluene was quantified using gas chromatography. Descriptive statistics were computed for the variables. Risk factors were evaluated using multiple logistic regression analysis. Adjusted odds ratio (ORadj) and 95\% confidence interval (CI) were presented as statistically significant when the $\mathrm{p}$ value was $<0.05$. The Mann-Whitney $\mathrm{U}$ test was used to compare the medians of continuous variables of the two groups. The prevalence of skin effects $(60.71 \%)$; respiratory tract irritation (49.29\%); nausea (46.43\%); and dizziness $(40.71 \%)$ was remarkable in the automotive garage workers. Several socio-demographic variables were significantly associated with increased skin effects, respiratory tract irritant, nausea, and dizziness. The median urinary toluene level of the automotive garage workers was $145 \mu \mathrm{g} / \mathrm{L}$ (range, 12.0$958.0 \mu \mathrm{g} / \mathrm{L}$ ) which the median urinary levels and demographic characteristics, occupational lifestyle, personal protective equipment used, hygiene behavior, and adverse health symptoms had significantly significant different $(\mathrm{p}<0.05)$.Urine is one of the most useful a sample for biomonitoring of occupational exposure to toluene. Personal hygiene is important for the automotive garage workers, and it should be emphasized in education programs.
\end{abstract}

Keywords: Urinary toluene levels, Adverse health symptoms, Automotive garage workers

\section{Introduction}

The current economic situation is contributing to the opening of garage businesses. In 2018-2020, domestic car sales are likely to expand continuously in line with the economic direction while cars aged 5 years or more will turn to general car repair services instead of going to the repair center more [1]. Automotive garage businesses perform repairs and maintenance, tapping, and painting of cars. Each of these processes use many organic chemicals and solvents in different stages, which cause automotive garage workers to be exposed to organic chemicals and solvents that are related to adverse health symptoms and can result in environmental contamination. Workers who work in automotive garages are exposed to dust, air pollutants [2], heavy metals [3,4], and organic solvents [5,6]. Exposure to different chemical substances in automotive garages may cause respiratory disorders in exposed workers. Diseases of the respiratory system induced by occupational dust, gas, and vapours, are influenced by the type of dust, gas, or vapour, as well as duration of exposure [7]. Some studies have shown a high prevalence of respiratory symptoms [8], cardiovascular health effects [9]. In addition, renal failure, encephalopathy, hepatotoxicity [10], liver and kidney [11,12], lung faction [13] can be seen among workers who were exposed to various toxicants from working as garage attendants. Besides, as reported, there were higher prevalence of selfreported chronic respiratory symptoms and dermal symptoms in workers inside the greenhouse compared to those in the controls [9]. Therefore, the different groups of auto-technicians came in contact with fuel while working, which may have numerous negative health consequences that include dermatitis, skin sensitization, eczema, and oil acne [14]. An automotive garage is undermined by poor working environments (i.e., dirty workplaces). Poor section workplace has been found across all sectors, with associated health hazards. Exposure to mixtures of organic solvents may be associated with the prevalence of hypertension in car-manufacturing workers [15]. In addition, factors influencing adverse health symptoms in workers may include long working hours [16], kind of personal protective equipment used, and habits, such as smoking, drinking, eating, chewing khat, and taking showers at work, respectively. Some studies have shown the variation in the level of 
toluene in each work location can be due to the number of productions, type of raw materials used, work methods, inadequate ventilation, and workstation either indoor or outdoor $[17,18]$. In addition, the lack of proper storage of waste materials and poor personal hygiene is causes of high levels of toluene in the work environment $[19,20]$.

The purpose of the present study was to determine their urinary toluene levels, to describe the workers demographic characteristics, occupational lifestyle, personal protective equipment used, personal hygiene and the prevalence of adverse health symptoms among automotive garage workers exposed to chemical substances in the automotive garages of Southern Thailand.

\section{Materials and Methods}

Study population and samples

The Ethics Committee of the Institute of Research and Development, Thaksin University, approved this research. This cross-sectional descriptive study was conducted between November 1, 2020 and December 31, 2020 in automotive garages located in three districts, including the Phipun, Chawang, and Tham Phannara districts in the Nakhon Si Thammarat province, Thailand were 30 automotive garages to determine the sample size by using the Krejcie \& Morgan formula [21]. A total of 28 automotive garages were selected using a purposive sampling method. All the automotive garages were small enterprises categorized by their service capacity of approximately 20-50 cars/month and the number of workers in each shop (5-12 workers). There were accepted to participate in the study. The participant automotive garage workers were recruited by purposive selection. A total of 140 (out of 230) of all the workers at these 28 automotive garages agreed to participate in the study. Inclusion criteria for the participant automotive garage workers were being 20-62 years old and in occupational contact with automotive garages for at least 1 year. Cooperative letters and informed verbal consent were obtained from all study participants.

\section{Sample collection}

Socio-demographics, adverse health symptoms, and personal hygiene behaviour were collected by a questionnaire. Five experts approved the validity of this instrument. The content of this instrument had a validity score of 0.89 and a Kuder-Richardson 20 (KR-20) reliability score $>0.94$. The questionnaire was conducted via face-to-face interview. Information on the following variables was collected: general information (gender, age, education, smoking status, and alcohol consumption), worker characteristics (duration of work in contact with automotive garages and days worked per week) and personal hygiene behaviour while working in automotive garages (Personal Protective Equipment used (PPE), consumption of food and/or beverages in the work area, whether hands were washed before lunch, and whether clothes were changed after work). Respondents were asked about the practices that they performed and the frequencies of those activities, which were categorized into 'sometimes' or 'always' and 'yes' or 'no'.

The occurrence of adverse health symptoms amongst the automotive garage workers was also observed. Adverse health symptoms included headache, dizziness, nausea, vomiting, coughing spasms, chest tightness, a sensation of being unable to breathe, progressive memory loss, fatigue, poor concentration, irritability, persistent headaches, muscular weakness ,redness and blisters, irritation of the nose and lower airways, feelings of intoxication and respiratory tract irritation [21]. The adverse health symptoms were noted either during the initial study time or during a 3 month recall period. Information was also collected among automotive garage workers by self-reported complaints and the diagnoses of consultant doctors. Respondents were asked about the occurrence of each adverse health symptom and were required to reply with 'yes' or 'no'.

\section{Urine samples collection}

Urine samples of the 140 participants were collected at the end of shift. Spot urine samples were collected at the end of shift after 2 days exposure. Urine samples were collected in polyethylene bottles, and they were stored at $-20{ }^{\circ} \mathrm{C}$ until analysis. Urinary toluene levels were analyzed by a gas chromatograph (GC) (Model GC-148; Shimadzu, Tokyo, Japan).

\section{Determination of urinary toluene levels}

Urine samples were analyzed within a few days with periodical vertexing for $2 \mathrm{~h}$ before analysis. Two $\mathrm{mL}$ of headspace was injected onto a $0.5 \mathrm{~mL}$ loop of the gas chromatograph [22]. Sodium chloride and toluene (99.9\%) were used. Stock solutions of each of the above organic compounds were prepared in methanol (Mallinckrodt Baker Inc., Phillipsburg, USA) at a concentration of $1000 \mathrm{mg} \mathrm{L}^{-1}$, and stored at $4{ }^{\circ} \mathrm{C}$ in sealed amber vial until use. gas chromatography technique using a DB-1 capillary column (30 $\mathrm{m}-0.53 \mathrm{~mm}$ inner diameter; J\&W Scientific) and flame ionization detector with an oven temperature of $200^{\circ} \mathrm{C}$, injector, and detector temperature of $250{ }^{\circ} \mathrm{C}$ and a helium flow rate of $10 \mathrm{~mL} / \mathrm{min}$. Calibration curves were obtained spiking blank urine samples with six different concentrations of each solvent (5 replicates per concentration), toluene between 92 and $560 \mu \mathrm{L} \mathrm{L}^{-1}$ when CAR-PDMS fibers were used. 


\section{Statistical analysis}

Data were collected by questionnaire and analysed using a software program. For descriptive statistics, percentages and frequency values were computed for the variables. Risk factors were evaluated using multiple logistic regression analysis. Adjusted odds ratio (ORadj) and 95\% confidence interval (CI) were presented as statistically significant when the $p$ value was $<0.05$. The Mann-Whitney $U$ test was used to compare the medians of continuous variables of the two groups.

\section{Results}

\section{Socio-demographics among automotive garage workers}

The study subjects consisted of 140 automotive garage workers from Southern Thailand. A substantial portion of the workers were older than 42 years of age (60.0\%). All workers were Buddhist. The largest group had less than a secondary school-level education (56.4\%). The subjects consisted of 98 smokers (70.00\%) and 42 non-smokers $(30.00 \%)$, and $88.6 \%$ disclosed they consumed alcohol.

The majority $(81.4 \%)$ of automotive garage workers worked more than 8 hours per day, worked 6 days per week (79.3\%), and worked for more than 16 years (57.9\%). Most subjects used neither cotton masks ( $51.4 \%$ ) nor gloves ( $70.7 \%)$ when doing their work. All subjects (100.0\%) washed their hands before lunch, but $47.1 \%$ of them did not use detergents when washing their hands. More than half (62.1\%) ate lunch in the working areas, and $82.9 \%$ of all subjects did not change their clothes after work every day (Table 1).

\section{Prevalence of health symptoms among automotive garage workers}

The prevalence of self-reported adverse health symptoms in the preceding 3 month is shown in (Table 2). The prevalence of skin effects, such as irritation, dermatitis, skin sensitization, eczema, oil acne, redness and blisters (60.71\%); respiratory tract irritation (49.29\%); nausea (46.43\%); and dizziness (40.71\%) was remarkable in the automotive garage workers. The different socio-demographic independent variables, including age, education level, smoking status, alcohol consumption, hours worked per day, days worked per week, duration of work, use of PPE and personal hygiene, and the relationship between these above symptoms is shown in Table 2 
Table 1. Subject socio-demographic characteristics among automotive garage workers.

\begin{tabular}{|c|c|c|}
\hline Characteristic & $\mathrm{n}=\mathbf{1 4 0}$ & $(\%)$ \\
\hline \multicolumn{3}{|l|}{ Gender } \\
\hline Male & 140 & 100 \\
\hline \multicolumn{3}{|l|}{ Age (yrs) } \\
\hline$\leq 42$ & 56 & 40.0 \\
\hline$>42$ & 84 & 60.0 \\
\hline \multicolumn{3}{|l|}{ Mean $\pm S D, 42.31 \pm 5.20 \mathrm{yrs}$} \\
\hline \multicolumn{3}{|l|}{ Education } \\
\hline$\leq$ Secondary school / vocational certificate or equivalent & 79 & 56.4 \\
\hline > Secondary school / vocational certificate or equivalent & 61 & 43.6 \\
\hline \multicolumn{3}{|l|}{ Behavioral } \\
\hline \multicolumn{3}{|l|}{ Smokes cigarettes } \\
\hline Yes & 98 & 70.0 \\
\hline No & 42 & 30.0 \\
\hline \multicolumn{3}{|l|}{ Drink alcohol } \\
\hline Yes & 124 & 88.6 \\
\hline No & 16 & 11.4 \\
\hline \multicolumn{3}{|l|}{ Occupational lifestyle } \\
\hline \multicolumn{3}{|l|}{ Hours worked per day } \\
\hline 8 & 26 & 18.6 \\
\hline$\geq 8$ & 114 & 81.4 \\
\hline \multicolumn{3}{|l|}{ Worked days per week } \\
\hline 6 & 113 & 79.3 \\
\hline$>6$ & 29 & 20.7 \\
\hline \multicolumn{3}{|l|}{ Duration of work (years) } \\
\hline 16 & 63 & 42.1 \\
\hline$>16$ & 77 & 57.9 \\
\hline \multicolumn{3}{|l|}{ Mean $\pm S D, 16.41 \pm 5.70$ yrs. } \\
\hline \multicolumn{3}{|l|}{ Max 32 yrs, Min 10 yrs. } \\
\hline \multicolumn{3}{|l|}{ Personal protective equipment } \\
\hline \multicolumn{3}{|l|}{ Cotton mask } \\
\hline Yes & 68 & 48.6 \\
\hline No & 72 & 51.4 \\
\hline \multicolumn{3}{|l|}{ Gloves } \\
\hline Yes & 99 & 70.7 \\
\hline No & 41 & 29.3 \\
\hline \multicolumn{3}{|l|}{ Personal hygiene } \\
\hline Washed hands before lunch & 140 & 100 \\
\hline \multicolumn{3}{|l|}{ Washed hands with detergents } \\
\hline Yes & 74 & 52.9 \\
\hline No & 66 & 47.1 \\
\hline \multicolumn{3}{|l|}{ Consumption of food and/or beverages in the work area } \\
\hline Yes & 87 & 62.1 \\
\hline No & 53 & 37.9 \\
\hline \multicolumn{3}{|l|}{ Whether clothes were changed after work } \\
\hline Yes & 24 & 17.1 \\
\hline No & 116 & 82.9 \\
\hline
\end{tabular}


Decharat I Urinary toluene levels and adverse health symptoms among automotive garage workers

Table 2. Prevalence (percent) of adverse health symptoms among automotive garage workers during the preceding 3 months $(\mathrm{n}=140)$.

\begin{tabular}{|c|c|c|}
\hline Parameter & Count $(n=140)$ & $(\%)$ \\
\hline Headache & 25 & 17.86 \\
\hline Dizziness & 57 & 40.71 \\
\hline Persistent headaches & 8 & 5.71 \\
\hline Nausea & 65 & 46.43 \\
\hline Vomiting & 9 & 6.43 \\
\hline Coughing spasms & 7 & 5.00 \\
\hline Chest tightness & 15 & 10.71 \\
\hline Respiratory tract & 69 & 49.29 \\
\hline Fatigue & 11 & 7.86 \\
\hline $\begin{array}{l}\text { Skin effects; irritation; dermatitis, skin } \\
\text { sensitization, eczema, oil acne, redness, } \\
\text { and blisters }\end{array}$ & 85 & 60.71 \\
\hline Progressive memory loss & 13 & 9.29 \\
\hline Poor concentration & 17 & 12.14 \\
\hline Irritability & 22 & 15.71 \\
\hline Muscular weakness & 8 & 5.71 \\
\hline Feelings of intoxication & 18 & 12.86 \\
\hline
\end{tabular}

Several socio-demographic variables were significantly associated with increased skin effects (Table 3), including age $\left(\mathrm{OR}_{\mathrm{adj}}=2.7 ; 95 \% \mathrm{CI}=1.01-4.93\right)$, smoking cigarettes $\left(\mathrm{OR}_{\mathrm{adj}}=2.3 ; 95 \% \mathrm{CI}=1.08-4.09\right)$, drinking alcohol $(\mathrm{OR}$ adj $=2.6 ; 95 \%$ $\mathrm{CI}=1.28-4.98)$, hours worked per day $(\mathrm{OR}$ adj $=2.5 ; 95 \% \mathrm{CI}=1.26-4.88)$, duration of work $(\mathrm{OR}$ adj $=2.5 ; 95 \% \mathrm{CI}=1.26-4.91)$, cotton mask use $\left(\mathrm{OR}_{\mathrm{adj}}=2.4 ; 95 \% \mathrm{CI}=1.21-4.83\right)$, glove use $(\mathrm{OR}$ adj $=2.7 ; 95 \% \mathrm{CI}=1.21-4.99)$, washing hands with detergent $\left(\mathrm{OR}_{\mathrm{adj}}=2.4 ; 95 \% \mathrm{CI}=1.15-4.63\right)$, consumption of food and/or beverages and/or smokes cigarettes in the work area $(\mathrm{OR}$ adj $=$ 2.3; $95 \% \mathrm{CI}=1.13-4.67$ ), and whether clothes were changed after work (ORadj $=2.3 ; 95 \% \mathrm{CI}=1.12-4.71)$, respectively. Additionally, age $\left(\mathrm{OR}_{\mathrm{adj}}=2.3 ; 95 \% \mathrm{CI}=1.12-5.08\right)$, smoking cigarettes (ORadj $\left.=2.4 ; 95 \% \mathrm{CI}=1.18-4.39\right)$, drinking alcohol $(\mathrm{ORad})$ $=2.5 ; 95 \% \mathrm{CI}=1.25-4.81)$, days worked per week $(\mathrm{OR}$ adj $=2.4 ; 95 \% \mathrm{CI}=1.25-4.89)$, duration of work $(\mathrm{OR}=2.5 ; 95 \% \mathrm{CI}=1.21$ $4.77)$, cotton mask use $(\mathrm{OR}$ adj $=2.6 ; 95 \% \mathrm{CI}=1.11-4.89)$, glove use (ORadj $=2.7 ; 95 \% \mathrm{CI}=1.20-4.91)$, washing hands with detergent $(\mathrm{OR}$ adj $=2.4 ; 95 \% \mathrm{CI}=1.10-4.89)$, consumption of food and/or beverages and/or smokes cigarettes in the work area $(\mathrm{OR}$ adj $=2.3 ; 95 \% \mathrm{CI}=1.18-4.77)$, and whether clothes were changed after work $(\mathrm{OR}$ adj $=2.3 ; 95 \% \mathrm{CI}=1.15-4.78)$ were significantly associated with increased respiratory tract irritation (Table 3).

The multiple variable logistic regression analysis, when controlling for age, smoking cigarettes, drinking alcohol, hours worked per day, duration of work, cotton mask use, glove use, washing hands with detergent, consumption of food and/or beverages and/or smokes cigarettes in the work area, and whether clothes were changed after work, showed that statistically significant risk factors related to skin effects amongst automotive garage workers were age $(\mathrm{OR}$ adj $=2.7 ; 95 \% \mathrm{CI}$ $=1.01-4.93)$, smoking cigarettes $\left(\mathrm{OR}_{\mathrm{adj}}=2.7 ; 95 \% \mathrm{CI}=1.08-4.09\right)$, drinking alcohol (ORadj $\left.=2.7 ; 95 \% \mathrm{CI}=1.28-4.98\right)$, hours worked per day $\left(\mathrm{OR}_{\mathrm{adj}}=2.5 ; 95 \% \mathrm{CI}=1.26-4.88\right)$, duration of work $(\mathrm{OR}$ adj $=2.5 ; 95 \% \mathrm{CI}=1.26-4.91)$, cotton mask use $(\mathrm{OR}$ adj $=$ $2.4 ; 95 \% \mathrm{CI}=1.21-4.83)$, glove use $(\mathrm{OR}$ adj $=2.7 ; 95 \% \mathrm{CI}=1.21-4.99)$, washing hands with detergent $(\mathrm{OR}$ adj $=2.4 ; 95 \% \mathrm{CI}=1.15-$ 4.63), consumption of food and/or beverages and/or smokes cigarettes in the work area (ORadj $=2.3 ; 95 \% \mathrm{CI}=1.13-4.67)$, and whether clothes were changed after work $\left(\mathrm{OR}_{\mathrm{adj}}=2.3 ; 95 \% \mathrm{CI}=1.12-4.71\right)$. Additionally, the statistically significant risk factors related to respiratory tract irritation amongst automotive garage workers were age (ORadj $=2.3 ; 95 \% \mathrm{CI}=1.12-5.08)$, smoking cigarettes $\left(\mathrm{OR}_{\mathrm{adj}}=2.4 ; 95 \% \mathrm{CI}=1.18-4.39\right)$, drinking alcohol $(\mathrm{OR}$ adj $=2.5 ; 95 \% \mathrm{CI}=1.25-4.81)$, days worked per week $\left(\mathrm{OR}_{\mathrm{adj}}=2.4 ; 95 \% \mathrm{CI}=1.25-4.89\right)$, duration of work $(\mathrm{OR}$ adj $=2.5 ; 95 \% \mathrm{CI}=1.21-4.77)$, cotton mask use $(\mathrm{OR}$ adj $=2.6 ; 95 \% \mathrm{CI}=1.11-$ 
4.89), glove use $\left(\mathrm{OR}_{\mathrm{adj}}=2.7 ; 95 \% \mathrm{CI}=1.00-4.91\right)$, washing hands with detergent $\left(\mathrm{OR}_{\mathrm{adj}}=2.4 ; 95 \% \mathrm{CI}=1.10-4.89\right)$, consumption of food and/or beverages and/or smokes cigarettes in the work area (ORadj $=2.3 ; 95 \% \mathrm{CI}=1.18-4.77)$, and whether clothes were changed after work $\left(\mathrm{OR}_{\mathrm{adj}}=2.3 ; 95 \% \mathrm{CI}=1.15-4.78\right)$.

Several socio-demographic variables were significantly associated with increased nausea (Table 4), including age $\left(\mathrm{OR}_{\mathrm{adj}}=2.5 ; 95 \% \mathrm{CI}=1.21-4.89\right)$, hours worked per day $\left(\mathrm{OR}_{\mathrm{adj}}=2.2 ; 95 \% \mathrm{CI}=1.16-4.57\right)$, days worked per week $(\mathrm{OR}$ adj $=2.4$; $95 \% \mathrm{CI}=1.79-2.79)$, cotton mask use (ORadj $=2.5 ; 95 \% \mathrm{CI}=1.18-4.98)$, consumption of food and/or beverages and/or smokes cigarettes in the work area $(\mathrm{ORadj}=2.5 ; 95 \% \mathrm{CI}=1.13-4.87)$, and whether clothes were changed after work $(\mathrm{OR}$ adj $=2.4 ; 95 \%$ $\mathrm{CI}=1.11-4.91)$. Finally, the variables that were significantly associated with increased dizziness (Table 4$)$ included age (ORadj $=2.4 ; 95 \% \mathrm{CI}=1.15-4.70)$, drinking alcohol $\left(\mathrm{OR}_{\mathrm{adj}}=2.2 ; 95 \% \mathrm{CI}=1.13-4.77\right)$, days worked per week $(\mathrm{OR}$ adj $=2.5 ; 95 \% \mathrm{CI}=1.99$ $3.04)$, cotton mask use (ORadj $=2.5 ; 95 \% \mathrm{CI}=1.12-4.85)$, consumption of food and/or beverages and/or smokes cigarettes in the work area $\left(\mathrm{OR}_{\mathrm{adj}}=2.2 ; 95 \% \mathrm{CI}=1.18-4.57\right)$, and whether clothes were changed after work $(\mathrm{OR}$ adj $=2.4 ; 95 \% \mathrm{CI}=1.18-4.99)$.

Additionally, the statistically significant risk factors related to nausea amongst automotive garage workers (Table 4) included age $\left(\mathrm{OR}_{\mathrm{adj}}=2.4 ; 95 \% \mathrm{CI}=1.19-4.87\right)$, hours worked per day $(\mathrm{OR}$ adj $=2.2 ; 95 \% \mathrm{CI}=1.11-4.79)$, days worked per week $\left(\mathrm{OR}_{\mathrm{adj}}=2.4 ; 95 \% \mathrm{CI}=1.67-2.99\right)$, cotton mask use $\left(\mathrm{OR}_{\mathrm{adj}}=2.4 ; 95 \% \mathrm{CI}=1.14-4.97\right)$, consumption of food and/or beverages and/or smokes cigarettes in the work area $\left(\mathrm{OR}_{\mathrm{adj}}=2.4 ; 95 \% \mathrm{CI}=1.15-4.99\right)$, and whether clothes were changed after work $(\mathrm{OR}$ adj $=2.3 ; 95 \% \mathrm{CI}=1.12-4.87)$. Finally, the variables that were significantly associated with increased dizziness (Table 4) included age $\left(\mathrm{OR}_{\mathrm{adj}}=2.3 ; 95 \% \mathrm{CI}=1.14-4.97\right)$, drinking alcohol $(\mathrm{OR}$ adj $=2.2 ; 95 \% \mathrm{CI}=1.12-4.61)$, days worked per week (ORadj $=2.5 ; 95 \% \mathrm{CI}=1.97-3.15)$, cotton mask use $(\mathrm{OR}$ adj $=2.4 ; 95 \% \mathrm{CI}=1.11-4.92)$, consumption of food and/or beverages and/or smokes in the work area $\left(\mathrm{OR}_{\mathrm{adj}}=2.3 ; 95 \% \mathrm{CI}=1.16-4.59\right)$, and whether clothes were changed after work $(\mathrm{OR}$ adj $=2.3 ; 95 \%$ $\mathrm{CI}=1.10-4.97)$. 
Decharat I Urinary toluene levels and adverse health symptoms among automotive garage workers Table 3. Prevalence of skin effects and respiratory tract irritant among automotive garage workers in the preceding 3 months.

\begin{tabular}{|c|c|c|c|c|c|c|c|c|c|}
\hline \multirow[b]{2}{*}{ Characteristics } & \multirow[b]{2}{*}{$\mathrm{n}=140$} & \multicolumn{4}{|c|}{ Skin effects $(n=85)$} & \multicolumn{4}{|c|}{ Respiratory tract irritant $(n=69)$} \\
\hline & & Count & $\begin{array}{c}\text { Prevalence } \\
(\%)\end{array}$ & $\begin{array}{c}\text { ORadj } \\
(95 \% \text { CI })\end{array}$ & P-value & Count & $\begin{array}{c}\text { Prevalence } \\
(\%)\end{array}$ & $\begin{array}{c}\text { ORadj } \\
(95 \% \text { CI })\end{array}$ & P-value \\
\hline \multicolumn{10}{|l|}{ Socio-demographic } \\
\hline \multicolumn{10}{|l|}{ Gender } \\
\hline Male & 140 & 85 & 100 & & & 69 & 100 & & \\
\hline \multicolumn{10}{|l|}{ Age (yrs) } \\
\hline$\leq 42$ & 54 & 26 & 48.15 & $\begin{array}{c}2.7 \\
(1.01-4.93)\end{array}$ & $<0.001^{*}$ & 18 & 33.33 & $\begin{array}{c}2.3 \\
(1.12-5.08)\end{array}$ & $<0.001^{*}$ \\
\hline$>42$ & 86 & 59 & 68.60 & 1.0 & & 51 & 59.30 & 1.0 & \\
\hline \multicolumn{10}{|l|}{ Education } \\
\hline $\begin{array}{c}\leq \text { Secondary school / vocational certificate } \\
\text { or equivalent }\end{array}$ & 79 & 48 & 60.76 & $\begin{array}{c}1.5 \\
(0.79-2.05)\end{array}$ & 0.314 & 35 & 44.30 & $\begin{array}{c}1.2 \\
(0.75-2.23)\end{array}$ & 0.225 \\
\hline $\begin{array}{c}\text { > Secondary school / vocational certificate } \\
\text { or equivalent }\end{array}$ & 61 & 37 & 60.66 & 1.0 & & 34 & 55.74 & 1.0 & \\
\hline \multicolumn{10}{|l|}{ Behavioral } \\
\hline \multicolumn{10}{|l|}{ Smokes cigarettes } \\
\hline Yes & 98 & 68 & 69.39 & $\begin{array}{c}2.3 \\
(1.08-4.09)\end{array}$ & $<0.001^{*}$ & 59 & 60.20 & $\begin{array}{c}2.4 \\
(1.18-4.39)\end{array}$ & $<0.001^{*}$ \\
\hline No & 42 & 17 & 40.48 & 1.0 & & 10 & 23.81 & 1.0 & \\
\hline \multicolumn{10}{|l|}{ Drink alcohol } \\
\hline Yes & 124 & 82 & 66.13 & $\begin{array}{c}2.6 \\
(1.28-4.98)\end{array}$ & $<0.001^{*}$ & 65 & 52.42 & $\begin{array}{c}2.5 \\
(1.25-4.81)\end{array}$ & $<0.001^{*}$ \\
\hline No & 16 & 3 & 18.75 & 1.0 & & 4 & 28.57 & 1.0 & \\
\hline \multicolumn{10}{|l|}{ Hours worked per day } \\
\hline 8 & 26 & 10 & 38.46 & $\begin{array}{c}2.5 \\
(1.26-4.88)\end{array}$ & $<0.001^{*}$ & 11 & 42.31 & $\begin{array}{c}1.5 \\
(0.23-0.79)\end{array}$ & 0.245 \\
\hline$\leq 8$ & 114 & 75 & 65.79 & 1.0 & & 58 & 50.88 & 1.0 & \\
\hline \multicolumn{10}{|l|}{ Occupational lifestyle } \\
\hline \multicolumn{10}{|l|}{ Days worked per week } \\
\hline 6 & 113 & 67 & 59.29 & $\begin{array}{c}1.4 \\
(1.09-1.99)\end{array}$ & 0.231 & 59 & 52.21 & $\begin{array}{c}2.4 \\
(1.25-4.89)\end{array}$ & $<0.001^{*}$ \\
\hline$>6$ & 27 & 18 & 66.67 & 1.0 & & 10 & 37.04 & 1.0 & \\
\hline
\end{tabular}


Environmental Analysis Health and Toxicology 2021, 36(3):e2021018

Table 3. (continued)

\begin{tabular}{|c|c|c|c|c|c|c|c|c|c|}
\hline Duration of wc & & & & & & & & & \\
\hline 16 & 63 & 27 & 42.86 & $\begin{array}{c}2.5 \\
(1.26-4.91)\end{array}$ & $<0.001^{*}$ & 25 & 39.68 & $\begin{array}{c}2.5 \\
(1.21-4.77)\end{array}$ & $<0.001^{*}$ \\
\hline$\leq 16$ & 77 & 58 & 68.24 & 1.0 & & 44 & 57.14 & 1.0 & \\
\hline \multicolumn{10}{|c|}{ Mean \pm SD, $16.41 \pm 5.70$ yrs. } \\
\hline \multicolumn{10}{|c|}{ Personal protective equipment } \\
\hline \multicolumn{10}{|c|}{ Cotton mask } \\
\hline Yes & 68 & 33 & 48.53 & $\begin{array}{c}2.4 \\
(1.21-4.83)\end{array}$ & $<0.001^{*}$ & 8 & 11.76 & $\begin{array}{c}2.6 \\
(1.11-4.89)\end{array}$ & $<0.001^{*}$ \\
\hline No & 72 & 52 & 72.22 & 1.0 & & 61 & 84.72 & 1.0 & \\
\hline \multicolumn{10}{|c|}{ Gloves } \\
\hline Yes & 99 & 44 & 44.44 & $\begin{array}{c}2.7 \\
(1.21-4.99)\end{array}$ & $<0.001^{*}$ & 32 & 32.32 & $\begin{array}{c}2.7 \\
(1.20-4.91)\end{array}$ & $<0.001^{*}$ \\
\hline No & 41 & 41 & 100.00 & 1.0 & & 37 & 90.24 & 1.0 & \\
\hline \multicolumn{10}{|c|}{ Personal hygiene } \\
\hline \multicolumn{10}{|c|}{ Washed hands before lunch } \\
\hline Yes & 140 & & & & & & & & \\
\hline \multicolumn{10}{|c|}{ Washed hands with detergents } \\
\hline Yes & 75 & 33 & 44.00 & $\begin{array}{c}2.4 \\
(1.15-4.63)\end{array}$ & $<0.001^{*}$ & 20 & 26.67 & $\begin{array}{c}2.4 \\
(1.10-4.89)\end{array}$ & $<0.001^{*}$ \\
\hline No & 65 & 52 & 80.00 & 1.0 & & 49 & 75.38 & 1.0 & \\
\hline \multicolumn{10}{|c|}{$\begin{array}{l}\text { Consumption of food, smokes cigarettes } \\
\text { and/or beverages in the work area }\end{array}$} \\
\hline Yes & 87 & 62 & 71.26 & $\begin{array}{c}2.3 \\
(1.13-4.67)\end{array}$ & $<0.001^{*}$ & 53 & 60.92 & $\begin{array}{c}2.3 \\
(1.18-4.77)\end{array}$ & $<0.001^{*}$ \\
\hline No & 53 & 23 & 43.40 & 1.0 & & 16 & 30.19 & 1.0 & \\
\hline \multicolumn{10}{|c|}{ Whether clothes were changed after work } \\
\hline Yes & 24 & 5 & 20.83 & $\begin{array}{c}2.3 \\
(1.12-4.71)\end{array}$ & $<0.001^{*}$ & 7 & 29.17 & $\begin{array}{c}2.3 \\
(1.15-4.78)\end{array}$ & $<0.001^{*}$ \\
\hline No & 116 & 80 & 68.97 & & & 61 & 52.59 & 1.0 & \\
\hline
\end{tabular}

*significantly at 0.05 
Decharat I Urinary toluene levels and adverse health symptoms among automotive garage workers Table 4. Prevalence of nausea and dizziness among automotive garage workers in the preceding 3 months.

\begin{tabular}{|c|c|c|c|c|c|c|c|c|c|}
\hline \multirow[b]{2}{*}{ Characteristics } & \multirow[b]{2}{*}{$n=140$} & \multicolumn{4}{|c|}{ Nausea(n=65) } & \multicolumn{4}{|c|}{ Dizziness $(n=57)$} \\
\hline & & Count & Prevalence (\%) & $\begin{array}{c}\text { ORadj } \\
(95 \% \mathrm{CI})\end{array}$ & $\mathrm{P}$ - value & Coun & Prevalence (\%) & $\begin{array}{c}\text { ORadj } \\
(95 \% \mathrm{CI})\end{array}$ & P-value \\
\hline \multicolumn{10}{|l|}{ Socio-demographic } \\
\hline \multicolumn{10}{|l|}{ Gender } \\
\hline Male & 140 & 65 & 100.00 & & & 57 & 100.00 & & \\
\hline \multicolumn{10}{|l|}{ Age (yrs) } \\
\hline$\leq 42$ & 54 & 20 & 37.01 & $\begin{array}{c}2.5 \\
(1.21-4.89)\end{array}$ & $<0.001^{*}$ & 15 & 27.78 & $\begin{array}{c}2.3 \\
(1.14-4.97)\end{array}$ & $<0.001^{*}$ \\
\hline$>42$ & 86 & 45 & 52.33 & 1.0 & & 42 & 48.84 & 1.0 & \\
\hline \multicolumn{10}{|l|}{ Education } \\
\hline $\begin{array}{c}\leq \text { Secondary school / vocational } \\
\text { certificate or equivalent }\end{array}$ & 79 & 32 & 40.51 & $\begin{array}{c}1.3 \\
(0.84-2.20)\end{array}$ & 0.308 & 30 & 52.63 & $\begin{array}{c}1.1 \\
(0.63-2.20)\end{array}$ & 0.298 \\
\hline $\begin{array}{c}\text { > Secondary school / vocational } \\
\text { certificate or equivalent }\end{array}$ & 61 & 33 & 54.10 & 1.0 & & 27 & 44.26 & 1.0 & \\
\hline \multicolumn{10}{|l|}{ Behavioral } \\
\hline \multicolumn{10}{|l|}{ Smokes cigarettes } \\
\hline Yes & 98 & 42 & 42.86 & $\begin{array}{c}1.3 \\
(0.79-1.51)\end{array}$ & 0.089 & 38 & 38.78 & $\begin{array}{c}1.3 \\
(0.55-1.79)\end{array}$ & 0.093 \\
\hline No & 42 & 23 & 54.76 & 1.0 & & 19 & 45.24 & 1.0 & \\
\hline \multicolumn{10}{|l|}{ Drink alcohol } \\
\hline Yes & 124 & 57 & 49.97 & $\begin{array}{c}1.3 \\
(0.70-1.55)\end{array}$ & 0.357 & 49 & 39.52 & $\begin{array}{c}2.2 \\
(1.12-4.61)\end{array}$ & $0.012^{*}$ \\
\hline No & 16 & 8 & 50.00 & 1.0 & & 8 & 50.00 & 1.0 & \\
\hline \multicolumn{10}{|l|}{ Occupational lifestyle } \\
\hline \multicolumn{10}{|l|}{ Hours worked per day } \\
\hline 8 & 26 & 6 & 23.08 & $\begin{array}{c}2.2 \\
(1.16-4.57)\end{array}$ & $<0.001^{*}$ & 9 & 34.62 & $\begin{array}{c}1.2 \\
(0.76-2.59)\end{array}$ & 0.059 \\
\hline$\leq 8$ & 114 & 59 & 51.75 & 1.0 & & 48 & 42.11 & 1.0 & \\
\hline \multicolumn{10}{|l|}{ Days worked per week } \\
\hline 6 & 111 & 38 & 34.23 & $\begin{array}{c}2.4 \\
(1.79-2.79)\end{array}$ & $<0.001^{*}$ & 32 & 28.32 & $\begin{array}{c}2.5 \\
(1.97-3.15)\end{array}$ & $<0.001^{*}$ \\
\hline$>6$ & 29 & 27 & 93.10 & 1.0 & & 25 & 92.59 & 1.0 & \\
\hline
\end{tabular}


Duration of work (years)

\begin{tabular}{|c|c|c|c|c|c|c|c|c|c|}
\hline & & & & $(0.71-1.69)$ & & & & & \\
\hline 16 & 59 & 34 & 57.63 & 1.0 & 0.254 & 26 & 41.27 & $\begin{array}{c}1.2 \\
(0.36-2.39)\end{array}$ & 0.358 \\
\hline$\leq 16$ & 81 & 31 & 38.27 & & & 31 & 40.26 & 1.0 & \\
\hline \multicolumn{10}{|l|}{ Mean \pm SD, $16.41 \pm 5.70$ yrs. } \\
\hline \multicolumn{10}{|l|}{ Personal protective equipment } \\
\hline \multicolumn{10}{|l|}{ Cotton mask } \\
\hline Yes & 68 & 17 & 25.00 & $\begin{array}{c}2.5 \\
(1.18-4.98)\end{array}$ & $<0.001^{*}$ & 12 & 17.65 & $\begin{array}{c}2.4 \\
(1.11-4.92)\end{array}$ & $<0.001^{*}$ \\
\hline No & 72 & 48 & 66.67 & 1.0 & & 45 & 62.50 & 1.0 & \\
\hline \multicolumn{10}{|l|}{ Gloves } \\
\hline Yes & 99 & 48 & 48.48 & $\begin{array}{c}1.4 \\
(0.61-1.72)\end{array}$ & 0.159 & 42 & 42.42 & $\begin{array}{c}1.3 \\
(0.57-1.79)\end{array}$ & 0.159 \\
\hline No & 41 & 17 & 41.46 & 1.0 & & 15 & 36.59 & 1.0 & \\
\hline \multicolumn{10}{|l|}{ Personal hygiene } \\
\hline \multicolumn{10}{|l|}{ Washed hands before lunch } \\
\hline Yes & 140 & & & & & & & & \\
\hline Washed hands with detergents & & & & $\begin{array}{c}1.2 \\
(0.56-1.82)\end{array}$ & & & & & \\
\hline Yes & 75 & 32 & 42.67 & 1.0 & 0.143 & 28 & 37.33 & $\begin{array}{c}1.3 \\
(0.49-1.79)\end{array}$ & 0.177 \\
\hline No & 65 & 33 & 50.77 & & & 29 & 44.62 & 1.0 & \\
\hline \multicolumn{10}{|l|}{$\begin{array}{l}\text { Consumption of food and/or } \\
\text { beverages in the work area }\end{array}$} \\
\hline Yes & 87 & 54 & 62.07 & $\begin{array}{c}2.5 \\
(1.13-4.87)\end{array}$ & $<0.001^{*}$ & 41 & 47.13 & $\begin{array}{c}2.3 \\
(1.16-4.59)\end{array}$ & $<0.001^{*}$ \\
\hline No & 53 & 11 & 20.75 & 1.0 & & 16 & 30.19 & 1.0 & \\
\hline \multicolumn{10}{|l|}{$\begin{array}{l}\text { Whether clothes were changed } \\
\text { after work }\end{array}$} \\
\hline Yes & 24 & 6 & 25.00 & $\begin{array}{c}2.4 \\
(1.11-4.91)\end{array}$ & $<0.001^{*}$ & 2 & 8.33 & $\begin{array}{c}2.3 \\
(1.10-4.97)\end{array}$ & $<0.001^{*}$ \\
\hline No & 116 & 59 & 50.86 & 1.0 & & 55 & 47.41 & 1.0 & \\
\hline
\end{tabular}

*significantly at 0.05 


\section{Urinary toluene levels among automotive garage workers, and demographic characteristics, occupational lifestyle, personal protective equipment used, hygiene behavior, and health symptoms among automotive garage workers}

The median urinary toluene level of the automotive garage workers was $145 \mu \mathrm{g} / \mathrm{L}$ (range, 12.0-958.0 $\mu \mathrm{g} / \mathrm{L}$ ). It was found that median urinary toluene levels and demographic characteristics (age and education level) had significantly significant different $(\mathrm{p}<0.05)$. Automotive garage workers who had older than 42 years of age had significantly higher urinary levels than those who had less than or equal 42 years of age $(p<0.05)$. Automotive garage workers who had less than a secondary school-level education had significantly higher urinary levels than those who had more than a secondary school-level education $(\mathrm{p}<0.05)$. Automotive garage workers who smokers had significantly higher urinary levels than those who did not smokers $(p<0.05)$. Automotive garage workers who had worked more than 8 hours per day had significantly higher urinary levels than those who had worked less than or equal 8 hours per day $(p<0.05)$. Automotive garage workers who had worked 6 days per week had significantly higher urinary levels than those who had worked less than or equal 6 days per week $(p<0.05)$. Automotive garage workers who had worked for more than 16 years had significantly higher urinary levels than those who had worked less than or equal 16 years $(\mathrm{p}<0.05)$. Automotive garage workers who used a mask, and/or wore gloves, had significantly lower urinary levels than those who did not ( $p<0.001$ for both). Automotive garage workers who ate snacks while working had significantly higher urinary levels than those who did not $(p<0.001)$. Automotive garage workers who did not used detergents when washing their hands had significantly higher urinary levels than those who used detergents $(p<0.001)$, and automotive garage workers who did not change their clothes after work every day had significantly higher urinary levels than those who change their clothes after work every day $(p<0.001)$. Automotive garage workers who reported symptoms of skin effects, such as irritation, dermatitis, skin sensitization, eczema, oil acne, redness, and blisters; respiratory tract irritation; nausea; and dizziness had significantly higher urinary levels than those who did not have symptoms ( $\mathrm{p}<0.001$ for all) (Table 5).

Table 5 Comparison between urinary toluene levels, and characteristics of automotive garage workers $(\mathrm{n}=140)$.

\begin{tabular}{|c|c|c|c|c|}
\hline \multirow[b]{2}{*}{ Characteristics } & \multirow[b]{2}{*}{$\mathrm{n}=140$} & \multicolumn{3}{|c|}{ Toluene in urine $(\mu \mathrm{g} / \mathrm{L})$} \\
\hline & & Median & $\begin{array}{l}\text { Interquartile range } \\
\text { (Range, min-max) }\end{array}$ & P-value \\
\hline \multicolumn{5}{|l|}{ Socio-demographic } \\
\hline \multicolumn{5}{|l|}{ Gender } \\
\hline Male & 140 & 145.00 & $531.0(946.0,12.0-958.0)$ & \\
\hline \multicolumn{5}{|l|}{ Age (yrs) } \\
\hline$\leq 42$ & 56 & 63.5 & $139.0(463.0,12.0-475.0)$ & $<0.001^{*}$ \\
\hline$>42$ & 86 & 582.0 & $739.0(946.0,12.0-958.0)$ & \\
\hline \multicolumn{5}{|l|}{ Education } \\
\hline $\begin{array}{c}\leq \text { Secondary school / vocational certificate } \\
\text { or equivalent }\end{array}$ & 79 & 587.0 & $622.0(946.0,12.0-958.0)$ & $<0.001^{*}$ \\
\hline $\begin{array}{c}>\text { Secondary school / vocational certificate } \\
\text { or equivalent }\end{array}$ & 61 & 52.0 & $67.5(463.0,12.0-475.0)$ & \\
\hline \multicolumn{5}{|l|}{ Behavioral } \\
\hline \multicolumn{5}{|l|}{ Smokes cigarettes } \\
\hline Yes & 98 & 405.0 & $673.0(946.0,12.0-958.0)$ & $<0.001^{*}$ \\
\hline No & 42 & 49.5 & $44.8(75.0,12.0-87.0)$ & \\
\hline \multicolumn{5}{|l|}{ Drink alcohol } \\
\hline Yes & 124 & 225.0 & $538.0(946.0,12.0-958.0)$ & 0.548 \\
\hline No & 16 & 220.5 & $524.0(946.0,14.0-958.0)$ & \\
\hline \multicolumn{5}{|l|}{ Occupational lifestyle } \\
\hline \multicolumn{5}{|l|}{ Hours worked per day } \\
\hline 8 & 26 & 26.0 & $45.0(75.0,12.0-87.0)$ & $<0.001^{*}$ \\
\hline$\geq 8$ & 114 & 225.0 & $529.5(946.0,12.0-958.0)$ & \\
\hline \multicolumn{5}{|l|}{ Days worked per week } \\
\hline 6 & 111 & 87.0 & $202.0(936.0,12.0-948.0)$ & $<0.001^{*}$ \\
\hline$>6$ & 29 & 687.0 & $375.0(946.0,12.0-958.0)$ & \\
\hline \multicolumn{5}{|l|}{ Duration of work (years) } \\
\hline 16 & 59 & 58.0 & $86.0(884.0,12.0-896.0)$ & $<0.001^{*}$ \\
\hline$\geq 16$ & 81 & 475.0 & $656.0(946.0,12.0-958.0)$ & \\
\hline
\end{tabular}


Table 5. (continued)

Personal protective equipment

Cotton mask
Yes
No
Gloves
Yes
No

$\begin{array}{cccc}68 & 54.0 & 53.0(313.0,12.0-325.0) & <0.001^{*} \\ 72 & 587.0 & 624.0(856.0,102.0-958.0) & \\ & & & \\ 99 & 62.0 & 134.0(936.0,12.0-948.0) & <0.001^{*} \\ 41 & 788.0 & 443.5(733.0,225-958.0) & \end{array}$

Personal hygiene

Washed hands with detergents

Yes

No

$74 \quad 56.0$

56.0

$72.0(313.0,12.0-325.0)$

$66 \quad 596.0 \quad 533.3(838.0,120.0-958.0)$

$<0.001^{*}$

Consumption of food, smokes cigarettes and/or

beverages in the work area

Yes $\quad 87 \quad 595.0$

$53 \quad 57.0$

$534.5(839.0,120.0-959.0)$

$<0.001^{*}$

No

$72.5(314.0,12.0-326.0)$

Whether clothes were changed after work

$\begin{array}{lcc}\text { Yes } & 24 & 55.0 \\ \text { No } & 116 & 590.0\end{array}$

$55.0 \quad 70.5(310.0,12.0-320.0)$

$<0.001^{*}$

Adverse health symptoms

Skin effects; irritation; dermatitis, skin sensitization, eczema, oil acne, redness and blisters

Yes
No

$\begin{array}{cccc}85 & 476.5 & 654.0(946.0,12.0-958.0) & <0.001^{*} \\ & 28.0 & 43.5(789.0,12.0-958.0) & \\ & & & \\ 69 & 587.0 & 640.0(871.0,87.0-958.0) & <0.001^{*} \\ & 56.0 & 65.3(789.0,12.0-801.0) & \\ 65 & 596.0 & 646.0(871.0,87.0-958.0) & <0.001^{*} \\ & 56.0 & 72.0(789.0,12.0-801.0) & \\ & & & \\ & & & \\ & 596.0 & 424.5(838.0,120.0-958.0) & <0.001 \\ & 58.0 & 88.0(789.0,12.0-801.0) & \\ & & & \end{array}$

${ }^{1}$ The statistical analysis is Mann - Whitney U Test.

${ }^{2 *}$ significantly at 0.05 .

\section{Discussion}

The results of this study show skin effects (such as irritation, dermatitis, skin sensitization, etc.) in $60.71 \%$ of automotive garage workers. This result is in line with many previous studies [24,25]. Automotive garage workers are exposed to different chemicals in their workplaces, which is supported by El-Saadawy MS et al. (2011) [26] who found garage workers are exposed to skin irritants in their workplaces [26-28], such as oils, greases, solvents, and detergents. Respiratory tract irritation was found in $49.29 \%$ of automotive garage workers, which is in line with a previous report [29] that reported workers exposed to VOCs presented lower levels of FVC, VC, and PEF than the control group, except FEV1/FVC\%, FEV1, FEF2575 and FEV1/VC\%. Automotive mechanics are also at increased risk for inhaling aromatic hydrocarbons, which can cause serious health issues in workers. In this study, automotive garage workers reported having nausea $(46.43 \%)$ and dizziness $(40.71 \%)$, which was supported by the WHO [30]. These toxic aromatic hydrocarbons may be dispersed during the production process, having effects on health and subsequent chronic effects of organic solvents on the central nervous system of exposed workers. The adverse health symptoms predominated in automotive garage workers over 42 years of age. Regarding the sociodemographic of this study, more than half of automotive garage workers (61.43\%) in this study were older than 42 years of age, with a duration of work $>16$ years in more than half $(55 \%)$.

In addition, the associations of adverse health symptoms with the period hours worked per day and days worked per week were supported by Wong et al. (2019) [31], who reported that the potential long weekly working hours and country of origin were shown to adversely affect the occupational health of workers. Many studies have shown the negative effects of long working hours on the risks of directly or indirectly [32], and significant decrease in physical activity for workers on 
Decharat I Urinary toluene levels and adverse health symptoms among automotive garage workers

overtime [16]. Thus, for automotive garage workers, longer working hours may expose them to more toxic materials during work [31].

Personal protective equipment (PPE) used among the automotive garage workers were statistically significantly associated with the prevalence of adverse health symptoms. In this study, cotton mask use and glove use were evaluated. The automotive garage workers who did not use PPE had a higher prevalence of adverse health symptoms when compared with the workers who used PPE. From previous research, the main reason for not using PPE (cotton mask and gloves) was found to be a lack of provision of the PPE by the owners of the garages and discomfort. This result was supported by Ataro et al. (2018) [33] who observed most participants (80\%) were found to be working without any proper PPE and use of PPE was found to be poor, with three workers using special shoes (boots), two workers using both gloves and a cotton mask, and one worker using a hat. Bull N, Riise T, Moen B. (2012) [34] showed that the subjects who used PPE had reductions in accidents and health effects at work. In this study, washing hands with detergent had an influence on reducing chemical contamination of the body, leading to a reduction in adverse health effects (such as skin irritation and respiratory tract irritation). This was supported by the WHO [35,36] that confirmed hand hygiene is the primary measure to reduce both infections and toxicity. The factors influencing behaviour depend on patterns of hand hygiene and self-protection. Besides, many chemical toxicants used in the automotive garage can be absorbed though the body, such as toluene, causing systemic toxicity by ingestion, inhalation, and being slowly absorbed through the skin [26-28, 37]. Thus, hand hygiene behaviour with detergents can reduce exposures to chemicals and reduce risk exposure.

Consumption of food and/or beverages and/or smoking cigarettes in the work area were statistically significantly associated with the prevalence of adverse health symptoms. This result was supported by the ATSDR [37], which confirmed a certain amount of a harmful chemical must enter your body. Harmful chemicals can enter the bodies of workers if they breathe, eat, or drink or if they are absorbed through their skin [26,27] [38-40]. Thus, a suggested way they can reduce their exposure (and that of their families) to chemicals at home, at work, and at play is to change clothes after work. In this study, the automotive garage workers who did not change their clothes after work had a higher prevalence of adverse health symptom when compared with the workers who changed their clothes after work. One hundred and nine of 140 automotive garage workers $(77.9 \%)$ had urinary toluene levels that exceeded the accepted safe standard $(30 \mu \mathrm{g} / \mathrm{L}$, biological exposure index) recommended by the American Conference of Governmental Industrial Hygiene (ACGIH) [41]. In this study we measured toluene levels in urine, because urinary toluene as best biomarkers of occupational exposure to toluene.

A present study found that many factors influence increased urinary toluene levels. Cigarette smoking enhanced elimination of toluene [42], and its relationship to urinary toluene levels showed statistically significant difference between smokers and non-smokers. EPA [42], reported the highest concentrations of toluene usually occur in indoor air from the use of common household product, and cigarette smoking. However, the present study differs from Decharat S [43], who showed no statistically significant difference between the exposed and non-exposed group. With regard to working duration, it was found the median urinary toluene levels differed significantly. This is supported by Hormozi et al [44], who reported a significant correlation between working years in the printing industry and urinary levels of $\mathrm{HA}(\mathrm{r}=0.363$, $\mathrm{P}=$ $0.02)$ in the exposed group.

Toluene is also flammable, and its vapor can be ignited by flames spars or other ignition source. The automotive garage workers can be exposed to toluene by breathing, swallowing, getting it on their skin or into their eyes. In this study found that automotive garage workers who used a mask and/or gloves, had significantly lower urinary toluene levels compared to those who did not. The author noted that the types of PPE in use in these automotive shops were inappropriate for this type of work. Most automotive garage workers used a cotton mask to protect themselves during work. Toluene may enter a cotton mask and penetrate a worker's airway. Automotive garage workers using these inappropriate protective devices may also mistakenly believe that they are protected. Thus, the employers identify all the potential safety hazards and choose the proper PPE and correspond to the nature of their work. This guideline recommended by the CDC [45]" that shown in page 13/16.

The results presented that automotive garage workers who had poor protective practices (such as did not use detergents when washing their hands, ate lunch in the working areas, and did not change their clothes after work every day) had a urinary toluene level up to $958.0 \mu \mathrm{g} / \mathrm{L}$ (range, 946.0, 12.0-958.0). These automotive garage workers normally did not use a cotton mask and gloves and had poor personal hygienic practice and was therefore the highest exposed workers of the group [43]. This result supported by the ATSDR [46], that recommended persons whose clothing or skin is contaminated with liquid toluene can cause secondary contamination by direct contact or through off gassing vapor. Thus, work environments should be made safe, favorable and conducive to enhance productivity and economic prosperity for both employer and employee. This result was supported by the Kuranchie FA et al [47].

A limitation of this study is that automotive garage workers' exposure to mix chemicals, although this study is sp ecified for toluene exposure. Thus, future study suggests the evaluation the chemical co-exposure. In addition, the author did not control the external factor of the occurrence of adverse health symptoms which the weakness in this research.

The training program is a critical tool in reducing occupational health disparities such as a program designed to teach automotive garage workers about the chemical hazards present in their workplace, etc. This concept was supported 
by the $\mathrm{O}^{\prime}$ Connor, $\mathrm{T}$ et al [48], who presents that worker can implement what they learn, is essential if trainings hope to have an impact on health and safety outcomes or workplace practices.

\section{Conclusions}

Automotive garage workers are exposed to toluene. Urine is one of the most useful ways for biomonitoring of occupational exposure to toluene [42]. This compound presents a good correlation with the level of exposure. In this study, air samples were not collected, so this may be a disadvantage in this research. In addition, the research area was partially selected. Thus, the sample group in this research may therefore be small group. At the same time, demographic characteristics (age and education levels), behavioral (smoked cigarettes), occupational lifestyle (hours worked per day, days worked per week, and duration of work), personal protective equipment (cotton mask and gloves used), and personal hygiene (washed hands with detergents, consumption food in the area, and whether clothes were changed after work) are important for the automotive garage workers, and it should be emphasized in education programs.

\section{Acknowledgement}

The authors thank among automotive garage workers in Nakhon Si Thammarat Province, Thailand. The authors also thank the Faculty of Health and Sports Science, Thaksin University, who supported this research.

\section{Conflict of interest}

No potential conflict of interest relevant to this article was reported.

\section{ORCID}

Somsiri Decharat:0000-0001-7621-2008

\section{References}

[1] Yongpisaphop W. Business/Industry Outlook 2018-2020 (Thai). Assessed on Dec 12, 2020. https://www.krungsri.com/bank/getmedia/d82a0182-d4e9-4465-ac2c-345b41dd3323/IO_Automobile 2018

[2] Obianime AW, Odili O, Olorunfemi OJ, Wokoma TO, Chuemere AN. Toxic air and soil in automobile workshop impact negatively on the health status of automechanics: the Nigeria environment. Int J Pharm Pharmacol 2017;1(3):1-13. https://doi.org/10.31531/2581-3080.1000111

[3] Ishola AB, Okechukwu IM, Ashimedua UG, Uchechukwu D, Michael EA, Moses O, et al. Serum level of lead, zinc, cadmium, copper and chromium among occupationally exposed automotive workers in Benin City. Int J Environ Pollut Res 2017;5(1):70-79.

[4] Alli LA. Blood level of cadmium and lead in occupationally exposed persons in Gwagwalada, Abuja, Nigeria. Interdiscip Toxicol 2015;8(3):146. http://doi.org/10.1515/intox-2015-0022

[5] Jo WK, Song KB. Exposure to volatile organic compounds for individuals with occupations associated with potential exposure to motor vehicle exhaust and/or gasoline vapor emissions. Sci Total Environ 2001;269(1-3):25-37. https://doi.org/10.1016/S0048-9697(00)00774-9

[6] Kamal A, Malik RN. Hematological evidence of occupational exposure to chemicals and other factors among auto-repair workers in Rawalpindi, Pakistan. Osong public health res perspect 2012;3(4):229-238.

https://doi.org/10.1016/j.phrp.2012.10.003

[7] Rodríguez E, Ferrer J, Zock JP, Serra I, Antó JM, Batle J, et al. Lifetime occupational exposure to dusts, gases and fumes is associated with bronchitis symptoms and higher diffusion capacity in COPD patients. PLoS one 2014;9(2):e88426. https://doi.org/10.1371/journal.pone.0088426

[8] MK KK, George LS. Pulmonary function of automobile repair workers in the informal sector of Raichur urban. Int J Community Med Public Health 2017;4(5):1510-1514. http://dx.doi.org/10.18203/2394-6040.ijcmph20171571

[9] Zhou HL, Jiang L, Mai SQ, Zhang JW, Wang Z, Rong X, et al. Analysis of the cardiovascular effects on workers expose to occupational benzene-toluene-xylene and noise in a painting workshop of an automobile company.Zhonghua lao dong wei sheng zhi ye bing za zhi= Zhonghua laodong weisheng zhiyebing zazhi= Chinese journal of industrial hygiene and occupational diseases 2019;37(4):280-283. https://doi.org/10.3760/cma.j.issn.1001-9391.2019.04.009

[10] Brautbar N, Williams J. Industrial solvents and liver toxicity: risk assessment, risk factors and mechanisms. Int J Hyg Environ Health 2002;205(6):479-491. https://doi.org/10.1078/1438-4639-00175 
Decharat I Urinary toluene levels and adverse health symptoms among automotive garage workers

[11] Ajani EO, Ajibola A, Salau BA, Odufuwa TK, Odewabi AO. Preliminary report on hepatic and cardiovascular risk assessment of automobile mechanics in Nigeria. Afr J biotechnol 2011;10(9):1705-1711.

https://doi.org/10.5897/AJB10.1629

[12] Soni K, Sharma RK, Gupta A, Yadav V. Impact of petroleum fumes on liver and kidney functioning of petrol filling attendants and garage attendants working in South Haryana, India. Eur J Pharm Med Res 2016;3(8):569-573.

[13] Nemer M, Kristensen P, Nijem K, Bjertness E, Skare Ø, Skogstad M. Lung function and respiratory symptoms among female hairdressers in Palestine: a 5-year prospective study. BMJ Open 2015;5(10):e007857. http://dx.doi.org/10.1136/bmjopen-2015-007857

[14] International Labour Organization. Encyclopaedia of occupational health and safety Vol.3. 4th ed. Geneva: International Labour Organization;1998.

[15] Attarchi M, Golabadi M, Labbafinejad Y, Mohammadi S. Combined effects of exposure to occupational noise and mixed organic solvents on blood pressure in car manufacturing company workers. Am J Ind Med 2013;56(2):243-251. https://doi.org/10.1002/ajim.22086

[16] Sato K, Hayashino Y, Yamazaki S, Takegami M, Ono R, Otani K, et al. Headache prevalence and long working hours: The role of physical inactivity. Public Health 2012;126(7):587-593. https://doi.org/10.1016/j.puhe.2012.02.014

[17] Azari MR, Hosseini V, Jafari MJ, Soori H, Asadi P, Mousavion SMA. Evaluation of occupational exposure of shoe makers to benzene and toluene compounds in shoe manufacturing workshops in east Tehran. Tanaffos 2012;11(4):43.

[18] Farshad A, Oliaei HK, Mirkazemi R, Bakand S. Risk assessment of benzene, toluene, ethylbenzene, and xylenes (btex) in paint plants of two automotive industries in iran by using the coshh guideline. European scientific journal 2013;3:270 276. https://doi.org/10.19044/esj.2013.v9n10p\%p

[19] Bunkuea K, Khamsean D, Upatcha P, Tangmandee T, Meevasana K. Occupational Exposure of Toluene in Wood Furniture Manufacturing Environment: A Case Study. Heal Environ J 2014;5:57-68.

[20] Maryiantari ES, Martiana T, Sulistyorini L. Analyze the Level of Health Risks from Exposure to Toluene in Shoes Craftsman Workers. Am Sci Res J Eng Technol Sci 2016;16(1):137-154.

[21] Krejcie RV, Morgan DW. Determining sample size for research activities. Educational and psychological measurement 1970;30(3):607-610. https://doi.org/10.1177/001316447003000308

[22] Alvarez-Leite EM, Duarte A, Barroca MM, Silveira JN. Possible effects of drinking and smoking habits on hippuric acid levels in urine of adults with no occupational toluene exposure. J Occup Health 1999;41(2):112-114.

https://doi.org/10.1539/joh.41.112

[23] ATSDR. Health Effects of Chemical Exposure. Assessed on Dec 12, 2020. https://www.atsdr.cdc.gov/emes/public/docs/health\%20effects\%20of\%20chemical\%20exposure\%20fs.pdf

[24] Warshaw EM, Hagen SL, Sasseville D, Maibach HI, DeKoven JG, Belsito DV, et al. Occupational contact dermatitis in mechanics and repairers referred for patch testing: retrospective analysis from the North American Contact Dermatitis Group 1998-2014. Dermatitis 2017;28(1):47-57. https://doi.org/10.1097/DER.0000000000000231

[25] Yakut Y, Uçmak D, Akkurt ZM, Akdeniz S, Palanci Y, Sula B. Occupational skin diseases in automotive industry workers Cutan Ocul Toxicol 2014;33(1):11-5. https://doi.org/10.3109/15569527.2013.787088

[26] El-Saadawy MS, Attwa EM, El-Tayeb IM, Zalat MM. Dermatoses and hematological disorders among car mechanics in Zagazig city and their effects on quality of life. Zagazig Univ Med J 2015;17(2):142-156.

[27] Sakhvidi MJZ, Loukzadeh Z, Tezerjani HD. Occupational hand dermatitis in car repair workers. AIMS public health 2019;6(4):577-586. https://doi.org/10.3934/publichealth.2019.4.577

[28] Zorba E, Karpouzis A, Zorbas A, Bazas T, Zorbas S, Alexopoulos, et al. Occupational dermatoses by type of work in Greece. Saf Health Work 2013;4(3):142-148. https://doi.org/10.1016/j.shaw.2013.06.001

[29] Harati B, Shahtaheri SJ, Karimi A et al. Evaluation of Respiratory symptoms among Workers in an Automobile Manufacturing Factory, Iran. Iran J Public Health 2018; 47(2): 237-245.

[30] World Health Organization.Chronic Effects of Organic Solvents on the Central Nervous System and Diagnostic Criteria. Vol. 5. World Health Organization. Copenhagen : World Health Organization, Regional Office for Europe ; Oslo : Nordic Council of Ministers, 1985.(Environmental Health). 
[31] Wong K, Chan AH, Ngan SC. The effect of long working hours and overtime on occupational health: a meta-analysis of evidence from 1998 to 2018. Int J environ res public health 2019;16(12):2102. https://doi.org/10.3390/ijerph16122102

[32] Messenger JC, Lee S, McCann D. Working time around the world. Trend in working hours, laws and policies in a global comparative perspective. International Labour Office; Geneva, Switzerland; 2007.

[33] Ataro Z, Geremew A, Urgessa F. Occupational health risk of working in garages: comparative study on blood pressure and hematological parameters between garage workers and Haramaya University community, Harar, eastern Ethiopia. Risk management and healthcare policy 2018;(11)35-44. https://doi.org/10.2147/RMHP.S154611

[34] Bull N, Riise T, Moen BE. Work-related injuries and occupational health and safety factors in smaller enterprises-a prospective study. Occup Med 2002;52(2):70-74. https://doi.org/10.1093/occmed/52.2.70

[35] The WHO. Guidelines on Hand Hygiene in Healthcare. Assessed on Dec 12, 2020. https://www.who.int/patientsafety/information centre/Last_April versionHH Guidelines\%5B3\%5D.pdf.

[36] ATSDR. Toxic Substances Portal - Toluene. Assessed on Dec 12, 2020. https://www.atsdr.cdc.gov/toxprofiles/tp.asp?id=161\&tid=29.

[37] Adela Y, Ambelu A, Tessema DA. Occupational lead exposure among automotive garage workers: a case study for Jimma town, Ethiopia. J Occup Med Toxicol 2012;7(1):1-8. https://doi.org/10.1186/1745-6673-7-15

[38] Attwa E, EI-Laithy N. Contact dermatitis in car repair workers. J Eur Acad Dermatol Venereol 2009;23(2):138-145. https://doi.org/10.1111/j.1468-3083.2008.02952.x

[39] Geraldine M, Venkatesh T. Evaluation,diagnosis, and treatment of lead poisoning in a patient with occupational lead exposure: a case presentation. J Occup MedToxicol 2007;2(1): 1-4. https://doi.org/10.1186/1745-6673-2-7

[40] CCOHS. How Workplace Chemicals Enter the Body. Assessed on Dec 15, 2020. https://www.ccohs.ca/oshanswers/chemicals/how chem.html.

[41] EPA. Toluene. Assessed on Dec 15, 2020. https://www.epa.gov/sites/default/files/2016-09/documents/toluene.pdf

[42] Decharat S. Hippuric acid levels in paint workers at steel furniture manufacturers in Thailand. Safety and health at work 2014; 5(1):227-233. https://doi.org/10.1016/j.shaw.2014.07.006

[43] Hormozi M, Mirzaei R, Nakhaee A, Payandeh A, Izadi S, Dehghan Haghighi J, et al. Quantification of urinary metabolites of toluene and xylene isomers as biological indices of occupational exposure in printing industry workers. Health Scope 2019;8(1):1-7. https://doi.org/10.5812/jhealthscpe.82962

[44] Occupational Health \& Safety. PPE the Automotive Industry Needs to Invest In. Assessed on Dec 15, 2020. https://ohsonline.com/Articles/2019/04/01/PPE-the-Automotive-Industry-Needs-to-Invest-In.aspx?Page=2.

[45] CDC. Managing Hazardous Materials Incidents Volume I, Emergency Medical Services U.S. Department of Human Services, Public Health Service,Agency for Toxic Substance and Disease Registry. https://wonder.cdc.gov/wonder/prevguid/p0000018/p0000018.asp

[46] Kuranchie FA, Angnunavuri PN, Attiogbe F, Nerquaye-Tetteh EN. Occupational exposure of benzene, toluene, ethylbenzene and xylene (BTEX) to pump attendants in Ghana: Implications for policy guidance. Cogent Environmental Science 2019; 5(1):1603418. https://doi.org/10.1080/23311843.2019.1603418

[47] O'Connor T, Flynn M, Weinstock D, Zanoni J. Occupational safety and health education and training for underserved populations. New Solutions: A Journal of Environmental and Occupational Health Policy 2014;24(1):83-106. https://doi.org/10.2190/NS.24.1.d 